\title{
Relationship between the Thermal Hardening of Ion-Implanted Resist and the Resist Removal Using Atomic Hydrogen
}

\author{
Takeshi Maruoka ${ }^{1, *}$, Yousuke Goto ${ }^{1}$, Masashi Yamamoto ${ }^{1}$, Hideo Horibe ${ }^{1,3}$, \\ Eiji Kusano $^{1}$, Kazuhisa Takao ${ }^{2}$ and Seiichi Tagawa ${ }^{3}$ \\ ${ }^{1}$ Research Laboratory for Integrated Technological Systems, Kanazawa Institute of Technology, \\ 3-1 Yatsukaho, Hakusan, Ishikawa 924-0838, Japan \\ ${ }^{2}$ Tokyo Ohka Kogyo Co., Ltd., \\ 7-8-16 Ichinomiya, Samukawa, Koza, Kanagawa 253-0111, Japan \\ ${ }^{3}$ The Institute of Scientific and Industrial Research, Osaka University, \\ 8-1 Mihogaoka, Ibaraki, Osaka 567-0047, Japan \\ E-mailhhoribe@neptune.kanazawa-it.co.jp
}

\begin{abstract}
Using atomic hydrogen for its excellent reduction ability, we demonstrated the removal rates and the hardness of ion-implanted resists. Removal rates of B-ion implanted resists using atomic hydrogen decreased with increasing implantation dose. According to the hardness of the resists, the indentation depth at 100mgf shifted toward the surface of the resists with increasing implantation doses. In contrast, removal rates of thermally hardened resists decreased with increasing baking temperatures. The indentation depth at $100 \mathrm{mgf}$ shifted toward the surface of the resists with increasing baking temperature. Ion-implanted resist removal rate using atomic hydrogen decreased compared with non-ion-implanted resist. We believe that this is because the resists were thermally hardened, attributable to the supplied energy from implanted B-ions.
\end{abstract}

Keywords: ion-implanted resist, atomic hydrogen, thermal hardening.

\section{Introduction}

In the semiconductor manufacturing process, semiconductor devices are formed on Si-wafer substrates using several processes (e.g., coating, resist patterning, etching, ion implantation, resist removal, and cleaning). Ion implantation is one of the most important processes in fabricating $\mathrm{p} / \mathrm{n}$ semiconductor devices. In the ion implantation process, a group of 13 or 15 elements (e.g., B, P, and As ions) are irradiated over the entire area of a substrate on which resist patterns have been fabricated. The ions are implanted into the resist, which can also play a role as a mask for the substrate. The ion-implanted resists are then heated to several hundred degrees Centigrade by energy supplied from the implanted ions [1]. It is difficult to remove the ion-implanted resist because the resist hardens and is denatured by the implanted ions [2]. The ion-implanted resist is actually removed by combining oxygen plasma ashing and chemicals such as a sulfuric acid and hydrogen peroxide mixture or an ammonia and hydrogen peroxide mixture, although the removal of the resist is troublesome at present, due to the cost of the chemicals and their disposal costs, as well as environmental burdens.

To overcome these problems, we examined ion-implanted resist removal using atomic hydrogen, which has excellent reduction ability. Resist removal using atomic hydrogen, which is a dry method without plasma, is expected to reduce costs and environmental burdens.

Ion-implanted resists (B-ion-implanted with a dose of $1 \times 10^{15}$ atoms $/ \mathrm{cm}^{2}$ ) have been removed using atomic hydrogen, but the resist removal rate was very low $(0.06 \mu \mathrm{m} / \mathrm{min})$ [3]. At the same time, 
for non-implanted resist (positive-tone novolak resist), removal rates exceeding $1 \mu \mathrm{m} / \mathrm{min}$ have been achieved using atomic hydrogen in the process [4]. Therefore, it may be possible to remove ion-implanted resists at a higher removal rate.

In this paper, we demonstrated the removal rates and the hardness of B-ion-implanted resists. The resist hardness was measured by nanoindentation [5]. Additionally, since we believe that thermal hardening is the mechanism for the hardening of ion-implanted resist, we also demonstrated the relationship between the removal rates of resists at various baking temperatures and the hardening of the resists.

\section{Experimental}

2.1. Preparation of the ion-implanted resists and the thermally hardened resists

A positive-tone novolak resist (AZ6112; AZ-Electronic Materials) was used for this work. The resist was spin-coated onto a $\mathrm{Si}$ wafer using a spin-coater (ACT-300A; ACTIVE) at 2000rpm for 20s. It was then pre-baked at $100^{\circ} \mathrm{C}$ for $1 \mathrm{~min}$ on a hotplate (digital hot plate, 720 series; PMC dataplate $\left.{ }^{\circledR}\right)$. B-ions were implanted into the resists at doses of $5 \times 10^{12}$ to $1 \times 10^{16}$ atoms $/ \mathrm{cm}^{2}$ with an acceleration energy of $70 \mathrm{keV}$, without wafer cooling. Table 1 lists the film thicknesses and implantation times for the ion-implanted resist. We also used thermally hardened resists, which were further baked at 100 to $400^{\circ} \mathrm{C}$ for $1 \mathrm{~min}$ after being pre-baked. Table 2 lists the film thickness of the thermally hardened resist at each baking temperature.

Table 1. Implantation doses, time, and film thickness of B-ion-implanted resists.

\begin{tabular}{ccc}
\hline $\begin{array}{c}\text { Implantation doses } \\
{\left[\text { atoms } / \mathrm{cm}^{2}\right]}\end{array}$ & $\begin{array}{c}\text { Implantation time Film thickness } \\
{[\mathrm{sec}]}\end{array}$ & {$[\mu \mathrm{m}]$} \\
\hline Non $($ Ref $)$ & - & 1.15 \\
\hline $5 \times 10^{12}$ & 33 & 0.84 \\
$5 \times 10^{13}$ & 51 & 0.85 \\
$5 \times 10^{14}$ & 320 & 0.85 \\
$5 \times 10^{15}$ & 2410 & 0.70 \\
$1 \times 10^{16}$ & 4940 & 0.68 \\
\hline
\end{tabular}

Table 2. Additional baking temperature and film thickness of thermally hardened resists.

\begin{tabular}{cc}
\hline Baking temperature $\left[{ }^{\circ} \mathrm{C}\right]$ & Film thickness $[\mu \mathrm{m}]$ \\
\hline Non (Ref) & 1.15 \\
100 & 1.10 \\
150 & 1.05 \\
200 & 0.95 \\
250 & 0.92 \\
300 & 0.83 \\
350 & 0.77 \\
400 & 0.69 \\
\hline
\end{tabular}

\subsection{Experiment setup}

Figure 1 presents a schematic diagram of the experiment apparatus. Atomic hydrogen was generated by contact catalysis on a tungsten (W) hot-wire catalyst. B-ion-implanted resists may be decomposed to volatile gases, such as methane $\left(\mathrm{CH}_{4}\right)$, methanol $\left(\mathrm{CH}_{3} \mathrm{OH}\right)$, [3] and diborane $\left(\mathrm{B}_{2} \mathrm{H}_{6}\right)$ by the reductive decomposition due to atomic hydrogen. We measured the resist removal times by visual observation from the outside of the chamber. The resist-removal rate was obtained by dividing the resist film thickness by the resist removal time. The sample size was $1 \mathrm{~cm}^{2}$. The hydrogen-nitrogen mixed gas $\left(\mathrm{H}_{2}: \mathrm{N}_{2}=10: 90 \mathrm{vol} \%\right)$ inflow rate was $300 \mathrm{sccm}$, the total pressure in the chamber was $21.3 \mathrm{~Pa}$ (the hydrogen pressure in the chamber was $2.13 \mathrm{~Pa}$ ), the distance from the top of the catalyst to the substrate was $20 \mathrm{~mm}$, and the hot catalyst temperature was $1950^{\circ} \mathrm{C} . \mathrm{N}_{2}$ gas was used to dilute the concentration of the $\mathrm{H}_{2}$ gas and avoid an explosion. It has been reported that the density of atomic nitrogen is less than $4 \times 10^{10} \mathrm{~cm}^{-3}$ when the catalyst temperature is $2770 \mathrm{~K}$. In contrast, the density of atomic hydrogen has been reported as $4 \times 10^{14} \mathrm{~cm}^{-3}$ at that temperature [6]. Therefore, the density of atomic nitrogen generated by a hot catalyst is very low compared with atomic hydrogen. Therefore, the atomic nitrogen might not influence the resist removal. In fact,

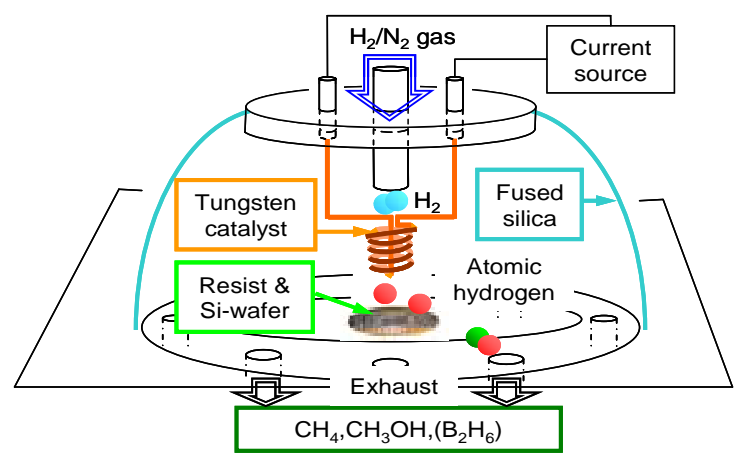

Fig. 1. Schematic diagram of the resist-removal apparatus using atomic hydrogen. 
the atomic nitrogen may be generated by a gas-phase reaction between the $\mathrm{N}_{2}$ and the atomic hydrogen. For example, $\mathrm{N}_{2}+\mathrm{H} \cdot \rightarrow \mathrm{H} \cdot+\mathrm{N} \cdot+\mathrm{N} \cdot$ and $\mathrm{N}_{2}+\mathrm{H} \cdot \rightarrow \mathrm{NH}+\mathrm{N} \cdot$. However, these reactions are difficult because the energy state of $\mathrm{N}_{2}+\mathrm{H} \cdot$ is lower than that of $\mathrm{H} \cdot+\mathrm{N} \cdot+\mathrm{N} \cdot$ and $\mathrm{NH}+\mathrm{N} \cdot[7]$.

\subsection{Nanoindentation test}

Figure 2 presents a schematic diagram of the apparatus use to evaluate the hardness of ion-implanted resist using nanoindentation (ENT-1040; ELIONIX). We examined the hardnesses at various resist depths by changing the maximum load from 1 to $260 \mathrm{mgf}$. The loading-unloading rate was set at $0.004 \mathrm{mgf} / \mathrm{ms}$ for a load of 1 to $8 \mathrm{mgf}$, and at $1 / 2000$ of the load for a load of more than $8 \mathrm{mgf}$. We evaluated the indenting hardness using a Berkovich-type diamond indenter with an apex angle of $115^{\circ}$.

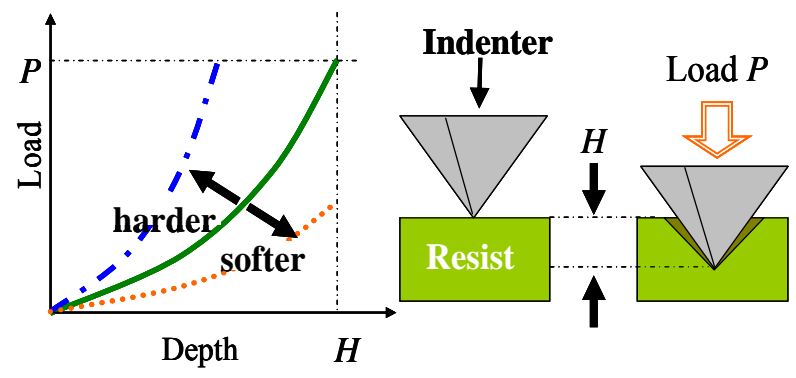

Fig. 2. Schematic diagram of the nanoindentation experiment apparatus.

\section{Results and Discussions}

3.1. Removal rate of B-ion-implanted resist and thermally hardened resist using atomic hydrogen

Figure 3 depicts the relationship between the removal rate of B-ion-implanted resist and the $\mathrm{B}$-ion implantation doses. The removal rates of the resists decreased with increasing implantation doses because the resists may thermally harden due

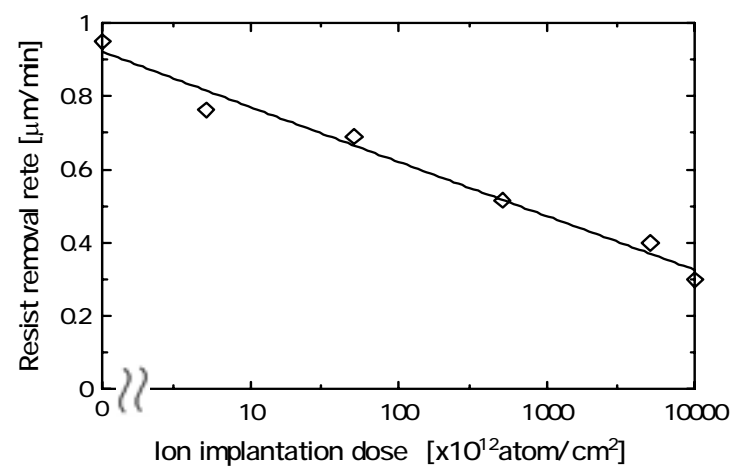

Fig. 3. Relationship between removal rate of B-ion-implanted resist and B-ion implantation dose. to the energy supplied from the implanted ions to the resist.

Figure 4 depicts the relationship between the removal rate of thermally hardened resists and the temperature of the additional baking. The resist removal rates decreased with increasing additional baking temperatures because the thermal energy increases with increasing temperature. Next, we will examine the hardness of the ion-implanted resists and the thermally hardened resists using nanoindentation.

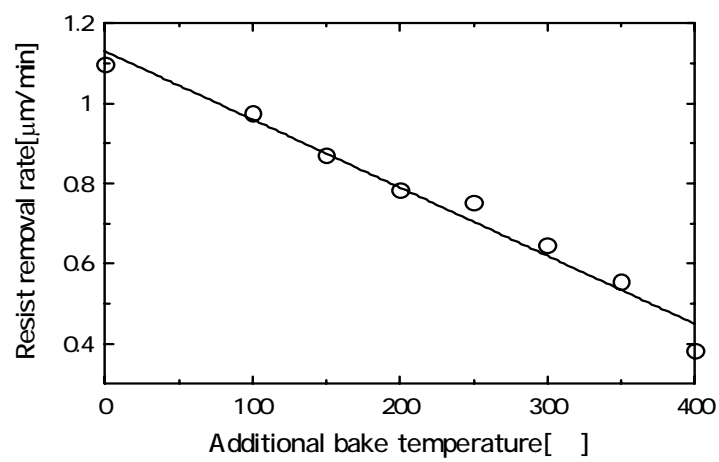

Fig. 4. Relationship between removal rates of thermally hardened resists and additional baking temperatures.

3.2. Hardness of ion-implanted resist and thermally hardened resist

Figure 5 depicts the change in indentation depth of the indenter with respect to several loads, for B-ion-implanted resists with doses from $5 \times 10^{12}$ to $1 \times 10^{16}$ atoms $/ \mathrm{cm}^{2}$. Table 3 lists the indentation depth of each ion-implanted resist at a load of 100 mgf. The indentation depth at 100mgf shifted toward the surface of the resists with increasing implantation doses. Therefore, the resists would thermally harden with increases in the implantation dose, because the energy supplied from the implanted ions to the resists increased with increasing implantation time.

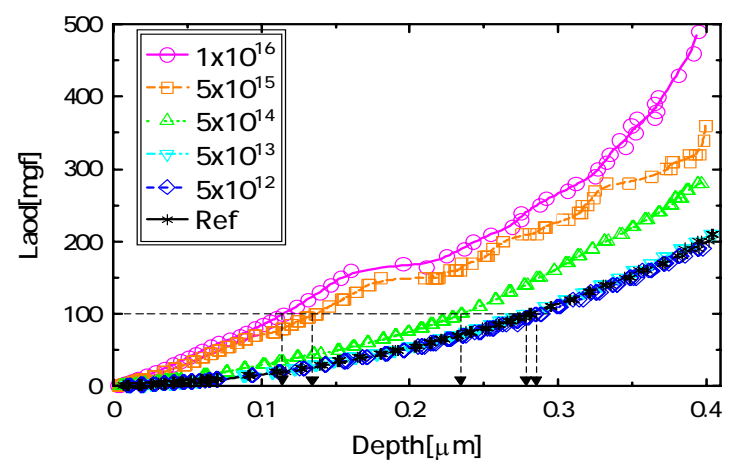

Fig. 5. Change of indentation depth of the indenter with several loads for resists with various B-ion implantation doses. 
Table 3. Indentation depth of each ion-implanted_resist with a load of $100 \mathrm{mgf}$.

\begin{tabular}{cc}
\hline Implantation doses [atoms $\left./ \mathrm{cm}^{2}\right]$ & Depth $[\mu \mathrm{m}]$ \\
\hline Non (Ref) & 0.28 \\
\hline $5 \times 10^{12}$ & 0.28 \\
$5 \times 10^{13}$ & 0.27 \\
$5 \times 10^{14}$ & 0.23 \\
$5 \times 10^{15}$ & 0.13 \\
$1 \times 10^{16}$ & 0.11 \\
\hline
\end{tabular}

Figure 6 depicts the indentation depth of B-ion implantation doses the indenter with respect to loads for thermally hardened resists that were additionally baked at 100 to $400^{\circ} \mathrm{C}$. Table 4 lists the indentation depth for each additional baking temperature at a load of 100mgf. The indentation depth at 100mgf shifted toward the surface of the resists with increasing additional baking temperature. Therefore, the resists would thermally harden with increasing baking temperature because the thermal energy increases with increasing temperature.

Consequently, the removal rates of ion-implanted resist by atomic hydrogen would decrease compared with non-ion-implanted resist, because the resist may thermally harden due to the supplied energy from the implanted ions.

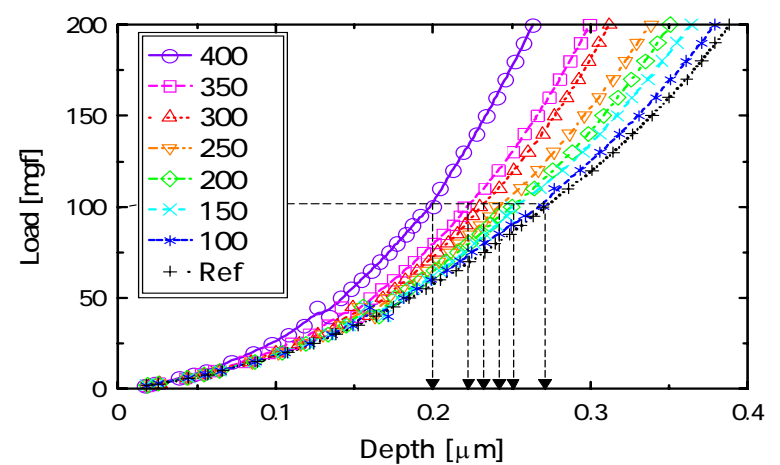

Fig. 6. Indentation depth of the indenter with respect to the loads for thermally hardened resists that were baked further at 100 to $400^{\circ} \mathrm{C}$.

Table 4. Indentation depth for each additional baking temperature at a load of $100 \mathrm{mgf}$.

\begin{tabular}{cc}
\hline Baking temperature $\left[{ }^{\circ} \mathrm{C}\right]$ & Depth $[\mu \mathrm{m}]$ \\
\hline Non (Ref) & 0.27 \\
100 & 0.27 \\
200 & 0.25 \\
250 & 0.25 \\
300 & 0.24 \\
350 & 0.23 \\
400 & 0.22 \\
\hline
\end{tabular}

\section{Conclusions}

Using atomic hydrogen for its excellent reduction ability, we demonstrated the removal rates and the hardness of B-ion-implanted resists. The resist hardness was measured by nanoindentation [5]. We also demonstrated the relationship between the removal rates of the resists at various baking temperatures and the hardening of the resists.

1) For B-ion-implanted resists, the resist removal rate decreased with increasing $\mathrm{B}$-ion implantation doses. The B-ion-implanted resist hardened with increases in implantation dose.

2) For thermally hardened resists, the resist-removal rate decreased with increasing additional baking temperature. The thermally hardened resist hardened with increasing temperature.

3) The removal rates of ion-implanted resist decrease with increasing implantation dose, because the resist may thermally harden due to the supplied energy from the implanted ions to the resist.

\section{Acknowledgments}

The authors are grateful to Professor Hideki Matsumura of the Japan Advanced Institute of Science and Technology for the idea of this atomic-hydrogen application. The authors are also grateful to Mr. Satoshi Ohya of Tokyo Ohka Kogyo Co., Ltd. Part of this study was supported by the Industrial Technology Research Grant Program (2004) from the New Energy and Industrial Technology Development Organization (NEDO) of Japan.

\section{References}

1. T. C. Smith, Handbook of Ion Implantation Technology, Ed. J. F. Ziegler, Elsevier Science Publishers B. V. (1992).

2. S. Fujimura, J. Konno, K. Hikazutani and H. Yano, Jpn. J. Appl. Phys., 28, 2130-2136

3. A. Izumi, H. Matsumura, Jpn. J. Appl. Phys. 41, 4640(2002).

4. K. Hashimoto, A. Masuda, H. Matsumura, T. Ishibashi and K. Takao, Thin Solif Films, 501, 326(2006).

5. A. Nakaue and N. Kawakami, Kobe Steel Engineering Reports, 52, 74-77(2002).

6. H. Umemoto, K. Ohara, D. Morimoto, M. Yamawaki, A. Masuda and H. Matsumura, Jpn. J. Appl. Phys., 42, 5315(2003).

7. P. J. S. B. Caridade, S. P. J. Rodrigues, F. Souda and A. J. C. Varandas, J. Phys. Chem., A 109, 2356(2005). 